\title{
Response of nuclear emulsions to ionizing radiations $\left({ }^{*}\right)$
}

\author{
R. KATZ and F. E. PINKERTON (**)
}

(Manuscrit reçu le 30 octobre 1975)

\begin{abstract}
Heavy ion tracks in Ilford $K$-2 emulsion are simulated with a computer program which makes use of the delta-ray theory of track structure, and the special assumption that the response of this emulsion to gamma-rays is 8-or-more hit. The Ilford $K$-series of nuclear emulsions is produced from a parent stock called $K .0$ emulsion, sensitized to become $K .1$ to $K .5$, and desensitized to become $K-1$ to $K-3$. Our simulations demonstrate that the emulsions $K .5$ through $K .0$ to $K-1$ are 1 -or-more hit detectors, while $K-2$ is an 8 -or-more hit detector. We have no data for $K-3$ emulsion. It would appear that emulsions of intermediate hittedness might be produced by an intermediate desensitization, to mimic or match the RBE-LET variations of biological cells, perhaps to produce a "rem-dosimeter". In the $K-2$ emulsion no developable grains are produced by stopping $\mathrm{H}, \mathrm{He}$, and $\mathrm{Li}$ ions. The emulsion has "threshold-like" properties resembling etchable track detectors. It should prove useful in the measurement of high LET dose in a strong low LET background, as for pions or neutrons. Since it can be expected to accumulate and repair "sub-lethal damage", to display the ion-kill and gamma-kill inactivation modes, the grain-count and track width regimes, it may serve to model biological effects.
\end{abstract}

\section{RÉSUMÉ}

On simule sur un ordinateur les traces d'ions lourds dans l'émulsion Ilford $K$-2 en utilisant un programme qui tient compte de la théorie de la structure des traces en fonction du rayonnement $\delta$ et avec l'hypothèse que la réponse de cette émulsion au rayonnement $\gamma$ est celle d'une cible à au moins huit coups.

(*) Presented at the 5th Symposium on microdosimetry, organized by the European Communities at Verbania-Pallanza, September 22-26, 1975.

Supported by the USERDA and the NSF (RANN).

(**) University of Nebraska, Lincoln NE 68508, U.S.A. 
Les émulsions Ilford $K$ sont obtenues à partir de l'émulsion $K .0$, sensibilisée pour donner les émulsions $K .1$ à $K .5$ et désensibilisée pour donner $K-1$ à $K-3$. Nos simulations montrent que les émulsions $K .5$ à $K-1$ sont des détecteurs à au moins un coup, tandis que $K-2$ est un détecteur à au moins huit coups. Nous n'avons pas de données sur l'émulsion $K-3$. Des émulsions à nombre de coups intermédiaire pourraient être obtenues, par une désensibilisation intermédiaire, pour correspondre aux variations EBR-TLE de cellules biologiques et, peut-être, conduire à un dosimètre-rem.

Dans l'émulsion $K$-2 les ions $\mathrm{H}, \mathrm{He}$ et $\mathrm{Li}$ ne produisent pas de grains développables. L'émulsion semble avoir un "seuil » comme les détecteurs ionographiques. Elle peut être utile pour la mesure de dose de rayonnement de TLE élevé dans un bruit de fond important de rayonnement TLE faible, comme des ions ou des neutrons. Une telle émulsion peut être utilisée comme modèle pour les effets biologiques puisqu'on peut espérer accumuler et réparer les dommages sub-létaux, présenter les modes d'inactivation des ions et des $\gamma$ et les régimes grains et traces.

\section{INTRODUCTION}

The response of many radiation detectors and biological systems to ionizing radiations is described by the delta-ray theory of track structure. Each detected end-point comes from a chain of events initiated by secondary and higher generation electrons which interact with sensitive sites within the targets. When the effect is measured as a function of the absorbed dose, differences in effect which come from differences in "radiation quality" are attributed to the spatial and temporal differences in the secondary electron distributions, on a scale comparable to the size and characteristic lifetimes of the targets. This model of a detector is patterned after a photographic emulsion, where the silver bromide crystals are the targets, or after biological tissue where the cell nuclei are the targets while some internal structures, possibly chromosomes, are the sensitive sites.

It has not been possible to predict detector response from first principles. Instead the theory assigns a set of operationally defined parameters to each detector. Once these are found experimentally, the formulas and concepts of the theory are used to predict detector response in any defined radiation environment [14].

The response of a detector to energetic heavy ions is calculated from its response to gamma-rays, and the radial distribution of local dose deposited around an ion's path by delta-rays. The two mathematical forms used to describe the response to gamma-rays, called the " multi-target single-hit ", or the "single-target multi-hit" models [5] give the probability $P$ that a target will be inactivated as multi-target single-hit :

$$
P(m, A)=\left(1-e^{-A}\right)^{m}
$$

and single-target multi-hit :

$$
P(c, A)=\sum_{x=c}^{\infty} \frac{A^{x} e^{-A}}{x !} .
$$




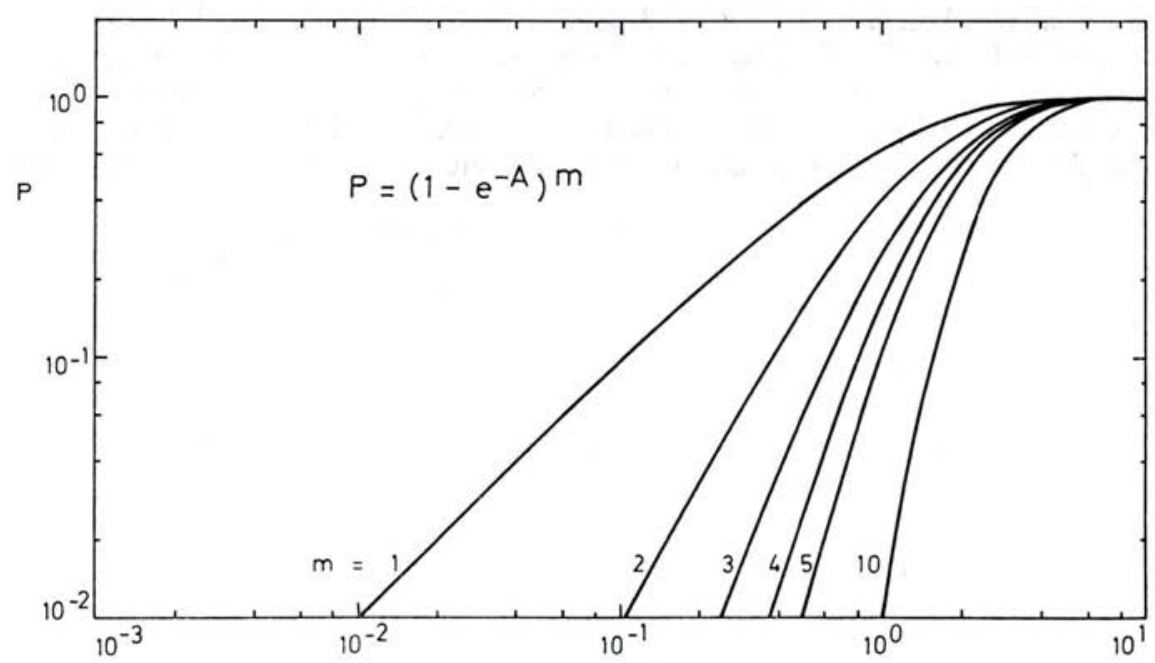

FIG. 1. - Inactivation probability $P$ vs. average number of hits per target, $A$, for the multi-target single-hit per target model, with $m$ sub-targets, or sensitive sites.

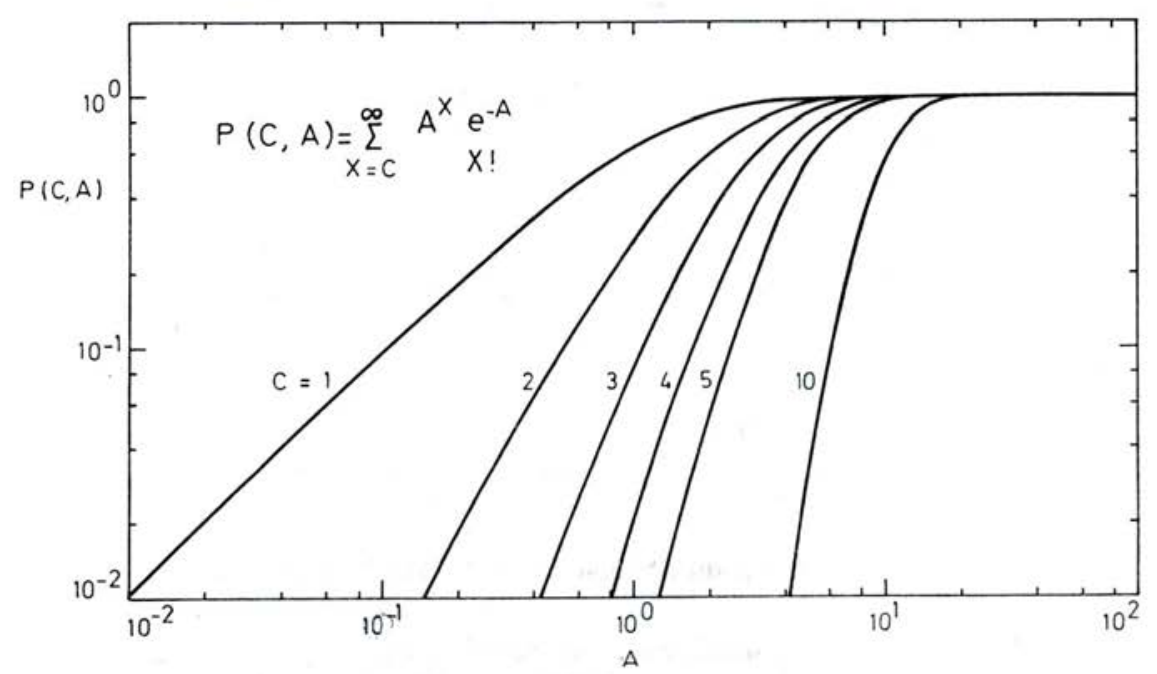

Fig. 2. $-P$ vs. $A$ for the multi-hit single-target model.

Here $m$ is the number of sensitive sites within a target, $c$ is the minimal number of hits required to inactivate a target, and $A=\bar{E} / E_{0}$ is the ratio of the average local dose $\bar{E}$ to a characteristic dose $E_{0}$ at which there is 1 hit per target. The two forms are identical when $m=c=1$ (1-hit detectors), are 
very similar when $m=c=2$ or 3 , and diverge at higher values, as shown in Figures 1 and 2. Physically a hit is interpreted to be a "registered event" caused by an electron passing through the sensitive site, with an efficiency depending on its speed. Most physical detectors are 1-hit detectors, where a single electron passing through a sensitive site either creates an observable

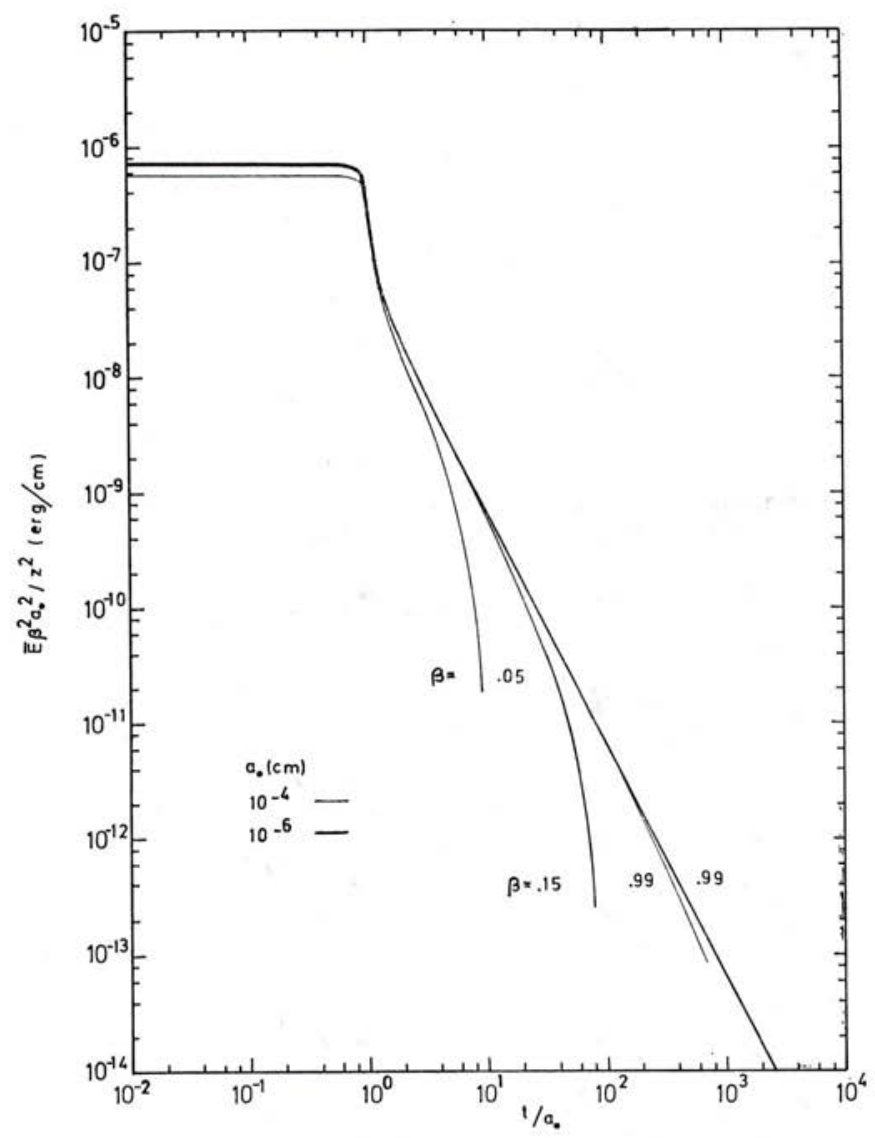

FIG. 3. - Radial distribution of local dose, in emulsion.

effect, or leaves the target unaltered. Otherwise, as in biological cells, more than one electron is required, and the detected end-point results from cumulative damage. The process then is much more complex, for there is a problem of the fading or repair of sub-lethal events, and the complication that heavily ionizing particles sometimes deposit sufficient energy (eject a sufficient number of delta-rays from the detector medium) to yield the required number of hits in the passage of a single particle (ion-kill) in a time shorter than atomic or molecular metastable lifetimes, and sometimes not (because of the statistical 
nature of the delta-ray production process) under apparently identical circumstances. In the latter case the detected end-point can only arise from the passage of many particles (gamma-kill). For the prediction of the response of the detector to heavy ions, we must first find the radial distribution of local dose about an ion's path, estimate the probability for ion-kill, and then (for detectors requiring more than 1-hit) accumulate the dose contribution of each particle to the gamma-kill mode of inactivation.

The radial distribution of the average local dose $\bar{E}\left(t, a_{0}\right)$ deposited in a representative target of radius $a_{0}$ whose center is at radial distance $t$ from the path of an ion of atomic number $Z$, effective charge number $z$, moving at relative speed $\beta$, through a medium containing $N$ electrons $/ \mathrm{cm}^{3}$, is found by first calculating the point-distribution of dose $E$ as

$$
E=\frac{N z^{2} e^{4}}{m c^{2} \beta^{2}}\left(\frac{1}{t^{2}}-\frac{1}{t \tau}\right),
$$

where we assume normal ejection of delta-rays, neglect binding energy, and take the electron to have constant energy loss, with the electron range $t$ given by $t=w k$, when its initial energy is $w$. The quantity $\tau$ is the range of the most energetic delta-ray from an ion moving at relative speed $\beta$, from kinematical considerations [4]. The point dose is averaged over a sensitive volume, approximated as a short cylinder whose axis is parallel to and at radial distance $t$ from the ion's path, with the result shown in Figure 3, for emulsion, where we have taken $N=1.04 \times 10^{24}$ electrons $/ \mathrm{cm}^{3}$, $k=3,015 \mathrm{~cm} / \mathrm{erg}$, and $a_{0}=0.12 \mu \mathrm{m}$, for the Ilford $K$-series [7]. More sophisticated calculations of $\bar{E}$ seem not to make substantial difference in the end result [12].

Once $\bar{E}$ is determined, and the dose-response function for the detector is known [say, Eqs. (1) or (2)], the radial distribution of inactivated elements (or sensitized emulsion grains) follows immediately. It is assumed that heavy ions generate delta-rays, that these produce higher generation electrons, and that the response of the medium to these is just as its response to gammarays except that the entire medium is more or less uniformly irradiated with gamma-rays, while there is a large local gradient in local dose about the path of each heavy ion, so that the detector must be examined in cylindrical shells about the ion's path.

The theoretical extension from single particle effects to the effects of beams of particles, or some complex radiation environments depends on the ability of the system to accumulate damage within a target.

\section{EXPERIMENT AND THEORY}

Experimental information dealing with the response of emulsions to ionizing radiations is sometimes available as blackness vs dose data (or vs incident fluence), and sometimes as particle track data, but both forms of data are typically not available from the same processed plate.

voL. $10-\mathrm{N}^{\circ} 4$ 
First, consider the blackness. If we take $G$ to be the number of grains per unit volume in the undeveloped emulsion, and $P$ to be the probability that a grain will become developed, the number of developed grains per unit volume is $P G$. In an emulsion layer of thickness $T$, the projected number of developed grains per unit area is $N=P G T$, and the measured blackness $B$, after processing is

$$
B=\frac{\alpha N \mathscr{A}}{2.3}
$$

where $\alpha$ is a factor intended to accomodate light scattering and optical differences among densitometers, and $\mathscr{A}$ is the cross-sectional area of the developed

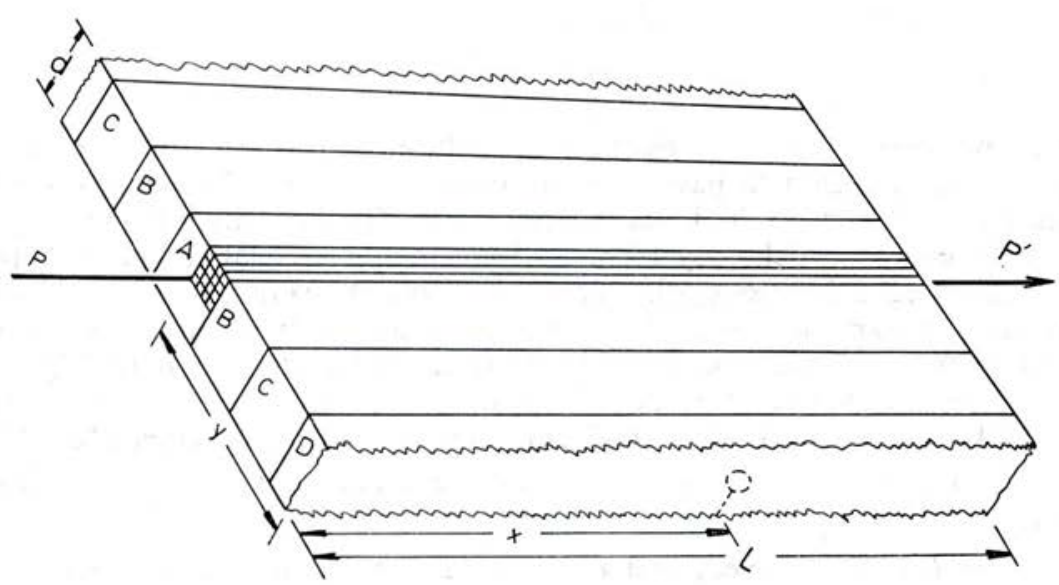

FIG. 4. - Box structure for computer simulation of particle tracks. The particle is taken to pass along $P P^{\prime}$. The depth of field is $d$, the segment length corresponding to a $\Delta \beta=0.01$ interval is $L$, while the placement of a dot at $x / L$ is determined by the output of a pseudorandom number generator. Boxes $A, B, B^{\prime}, C, C^{\prime}$ are centered on the raster lines of the visual display unit (a TV tube).

grain. For a 1-hit detector irradiated to dose $\bar{E}$, uniformly, with low LET radiations, we have

$$
B=\frac{\alpha G T \mathscr{A}}{2.3}\left(1-e^{-\bar{E} / E_{0}}\right),
$$

which describes the response of virtually (nearly) all emulsions to X-rays, gamma-rays, electron and even proton beams $[8,9,13,16,17]$. As for all 1-hit detectors there is a decline in radiosensitivity with an increase in the LET of the incident radiation, when the blackness is measured as a function of the macroscopic dose, for there is overkill at small $t$ adjacent to the path of 
K5

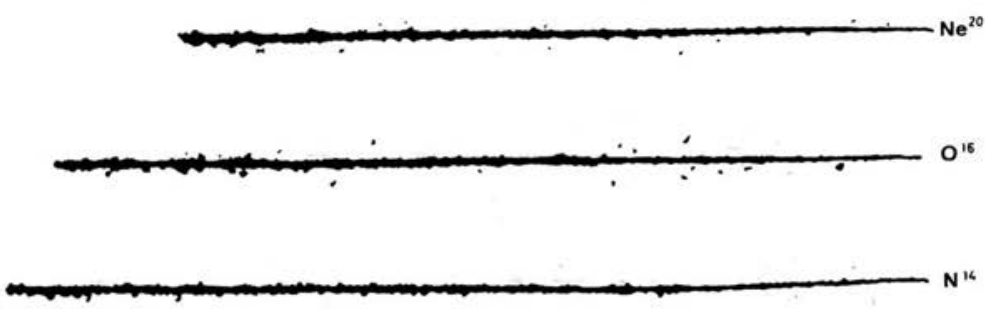

FIG. 5. - Tracks of ${ }^{40} \mathrm{~A},{ }^{20} \mathrm{Ne},{ }^{46} \mathrm{O},{ }^{14} \mathrm{~N}$, and ${ }^{12} \mathrm{C}$ ions in Ilford $K .5$ emulsion. Particles enter from left, at $10 \mathrm{MeV} / \mathrm{amu}$ and stop at right. Courtesy E.V. Benton.

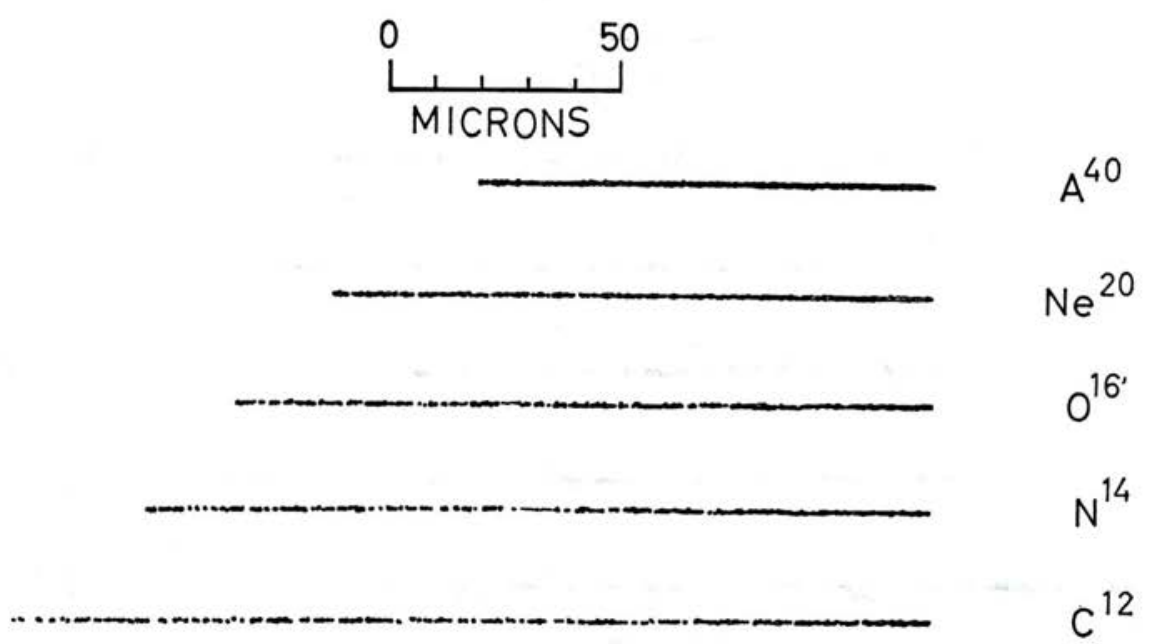

$C^{12}$

SIMULATED

FIG. 6. - Computer simulation of tracks in $K .5$ emulsion.

a heavy ion which is reflected in an apparently diminished value of $E_{0}$ as predicted by the theory. The radiosensitivity parameter $E_{0}$ is relatively independent of the energy of incident electrons or gamma-rays, when $\bar{E}$ is the absorbed dose in the emulsion, although there are large apparent variations

vOL. $10-\mathrm{N}^{\circ} 4$ 


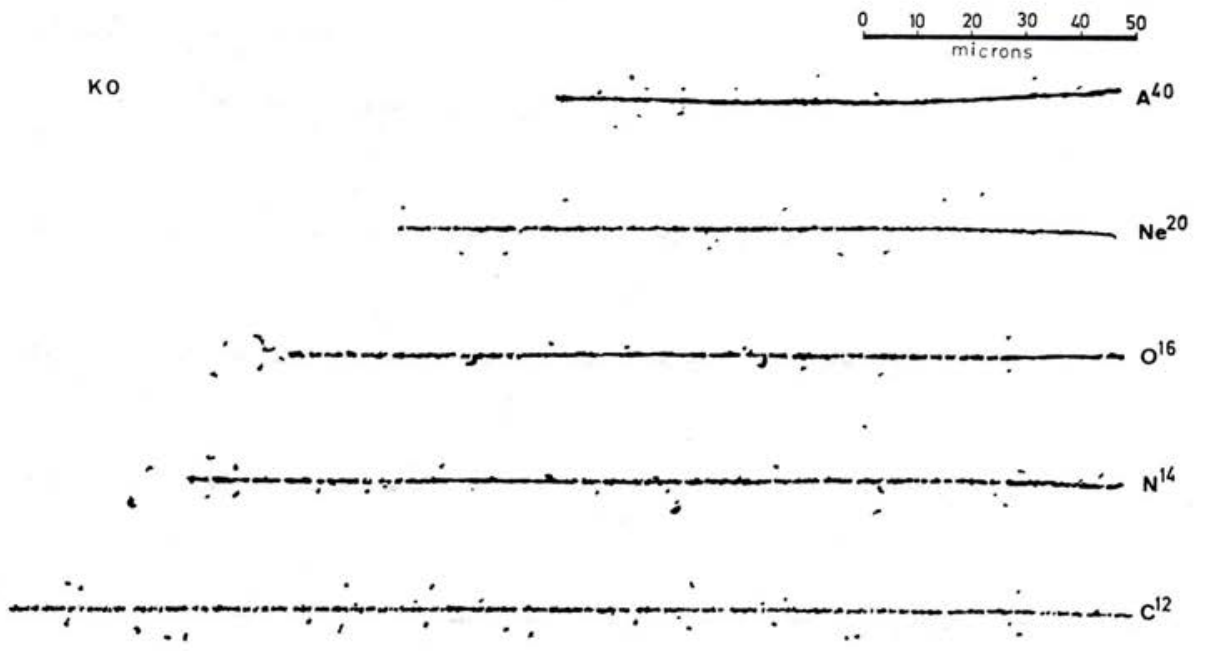

FIG. 7. - Tracks in Ilford K.0 emulsion. Courtesy E.V. Benton.
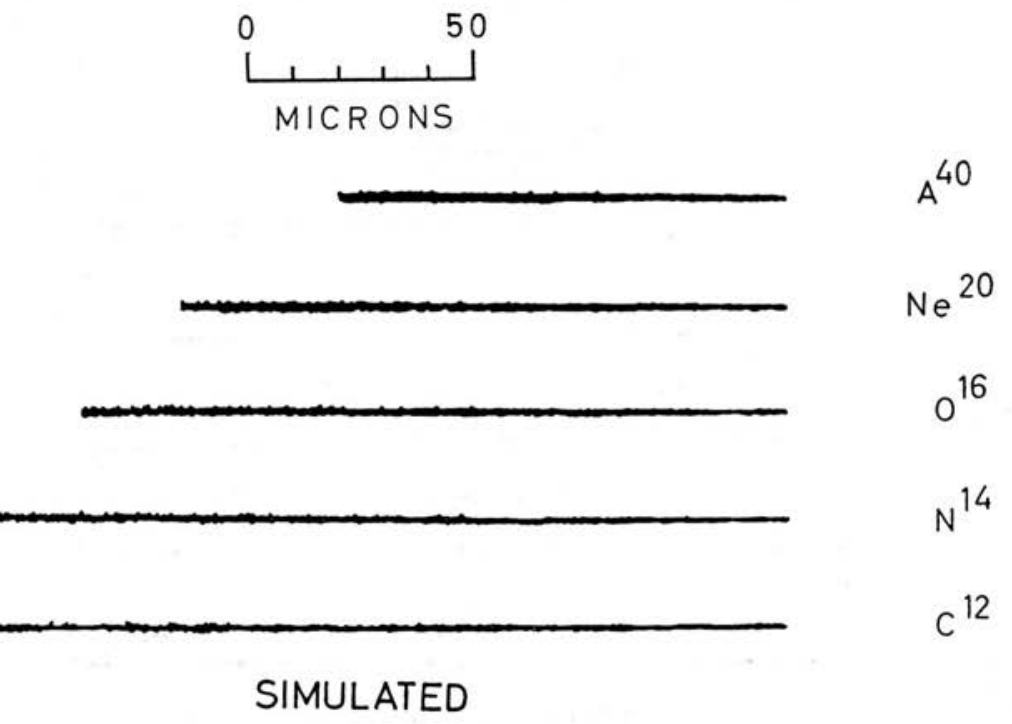

Fig. 8. - Computer simulation of tracks in $K .0$ emulsion.

in sensitivity with the energy of incident photons, when the blackness is plotted against the absorbed dose in a gaseous dosimeter [2]. There are suggestions in the literature of emulsions whose response to ionizing radiations is more than 1-hit $[2,6]$. In these cases a log-log plot of blackness $v s$. dose is not a line of unit slope but displays perhaps a greater slope, and some curvature. 


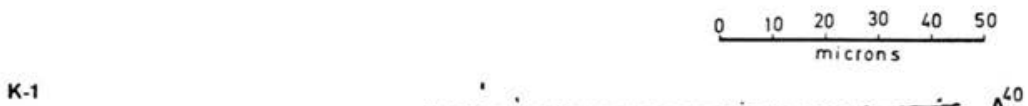

K-1 $A^{40}$

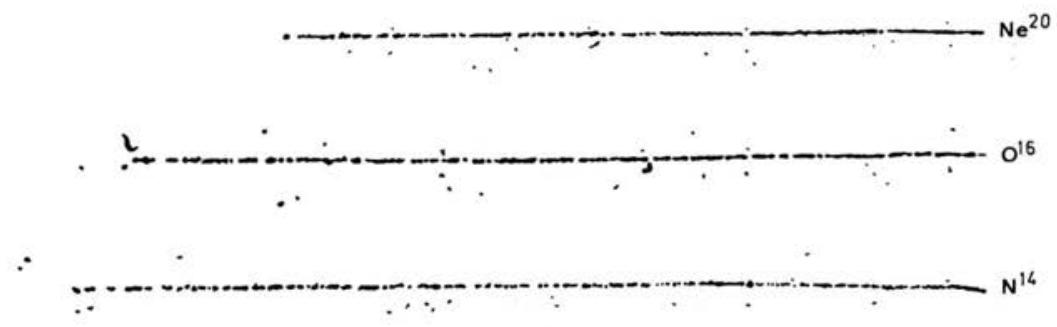
$c^{12}$

FIG. 9. - Tracks in Ilford $K-1$ emulsion. Courtesy E.V. Benton.

$\mathrm{K}-1$ $A^{40}$

$E_{0}=1,30 \times 10^{7}$ $\mathrm{Ne}^{20}$

$c=2$
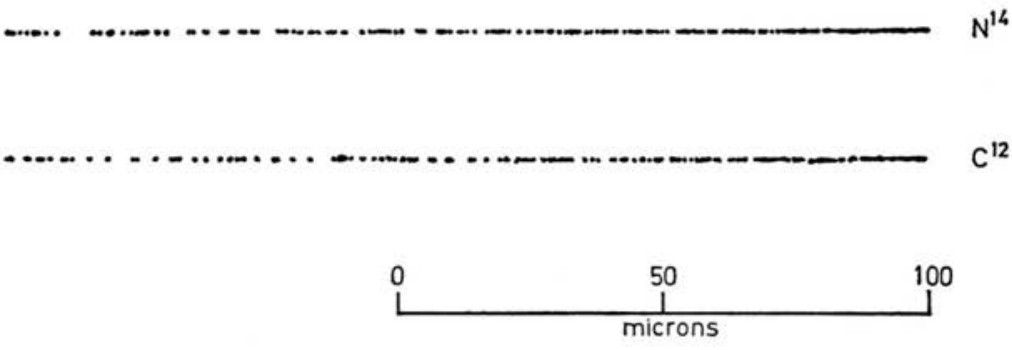

Fig. 10. - Computer simulation of tracks in $K-1$ emulsion, assuming it to be a 1-hit detector, like Figures 6 and 8 .

VOL. $10-\mathrm{N}^{\circ} 4$ 


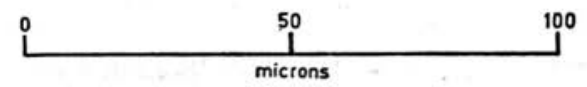

Fio. 11. - Computer simulation of tracks in $K-1$ emulsion, if it were a 2-or-more hit detector.

$\underbrace{\begin{array}{llllll}0 & 10 & 20 & 30 & 40 & 50\end{array}}_{\text {microns }}$

$\mathrm{K}-2$
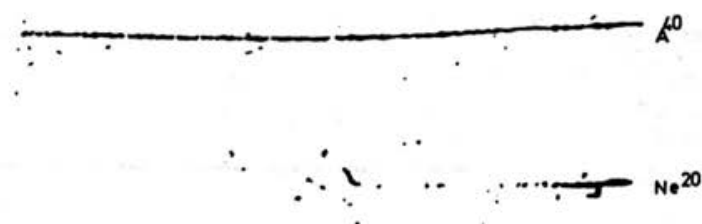

$\therefore$
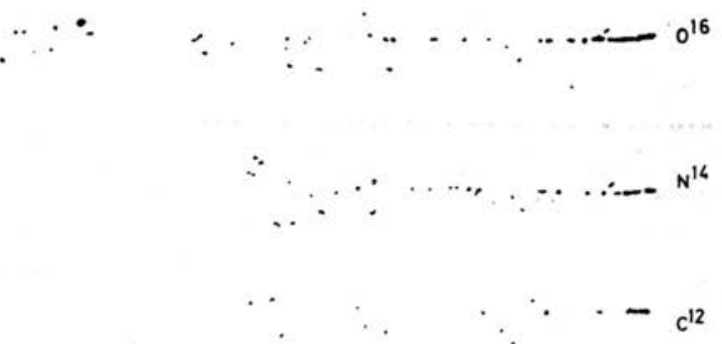

Fig. 12. - Tracks in Ilford K-2 emulsion. Courtesy E.V. Benton. 
Quantitative measurements of the tracks of individual particles in nuclear emulsion have also been consistent with the 1-hit assignment, and the requirements of the delta-ray theory of track structure. Measured values of the grain count in $G .5$ and $K .5$ emulsions, from energetic protons and heavier ions are in precise accord with the theory [12]. When microdensitometric measurements of the opacity structure of individual particle tracks are corrected
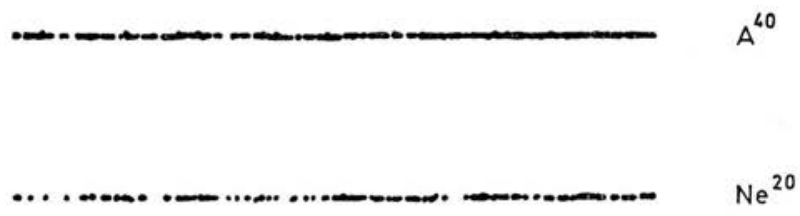

$K-2$

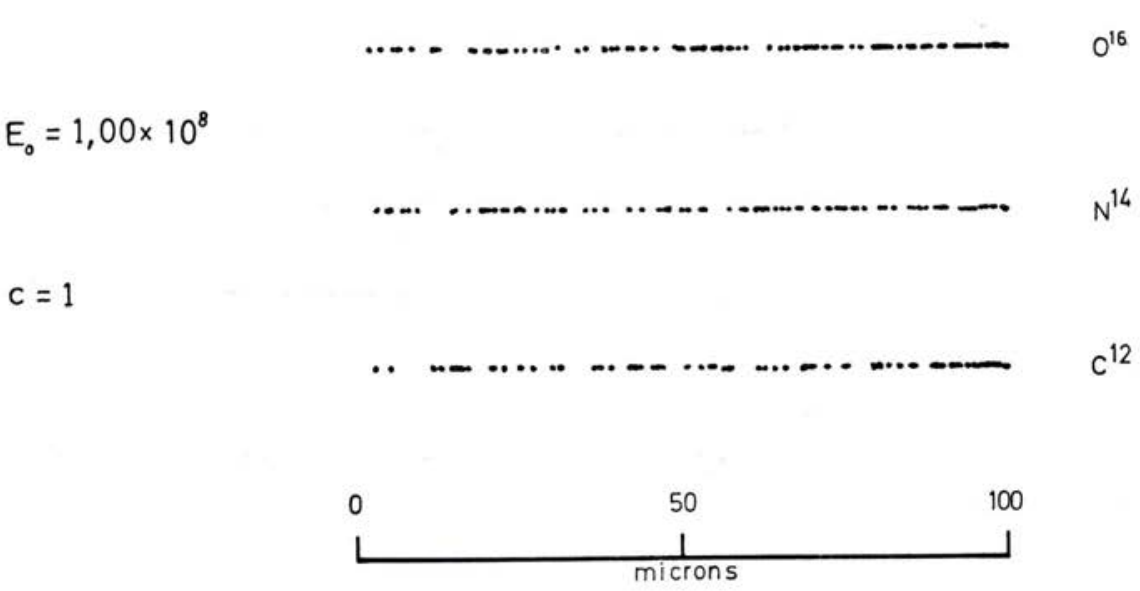

FIG. 13. - Attempted simulation of tracks in $K-2$ emulsion, if it were a 1-hit detector.

for optical problems in the microscope, the measurements are in excellent accord with the theory, for radial distances in emulsion from an ion's path from 0.5 to $40 \mu \mathrm{m}$, for relative speeds from 0.3 to 0.94 , and for ions of atomic number equal to or greater than $14[10,11]$.

Other data are available in the form of track photographs, of particles from accelerators, that are best treated as a problem in visual pattern recognition. To this end we have developed a computer program for the simulation of particle tracks in emulsion [7]. Developed grains are simulated as bright spots on the screen of an IBM 2250 visual display unit, are

voL. $10-\mathrm{N}^{\circ} 4$ 


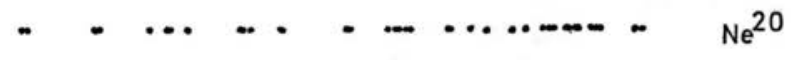

$K-2$

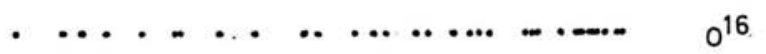

$E_{0}=4,00 \times 10^{8}$

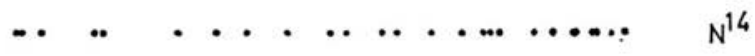

$c=1$

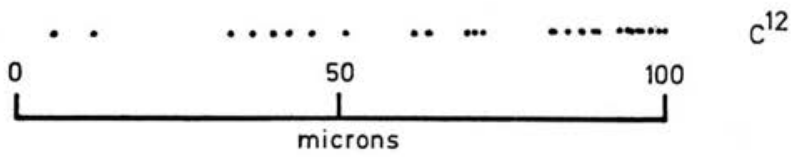

Fig. 14. - Second attempted simulation of tracks in $K-2$ emulsion, if it were a 1-hit detector.

$A^{40}$

$K-2$

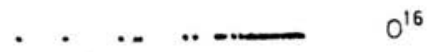

$E_{0}=8,00 \times 10^{6}$

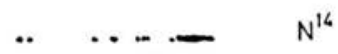

$c=8$

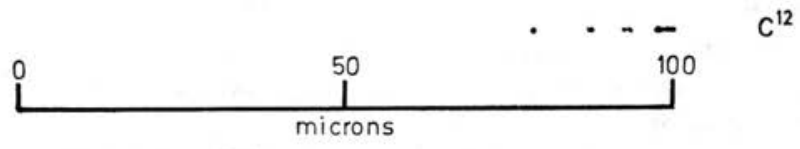

Fig. 15. - Simulation of tracks in $K-2$ emulsion, an 8-or-more hit detector. 

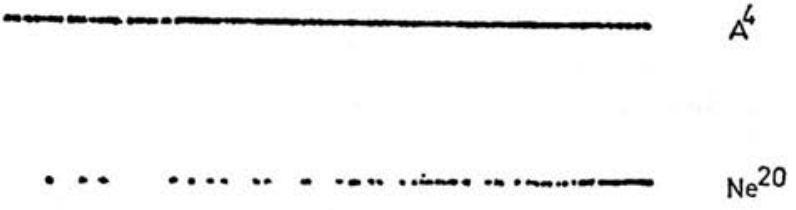

$\mathrm{K}-2$

$E_{0}=1,00 \times 10^{7}$

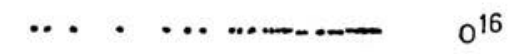

$c^{12}$

$c=6$

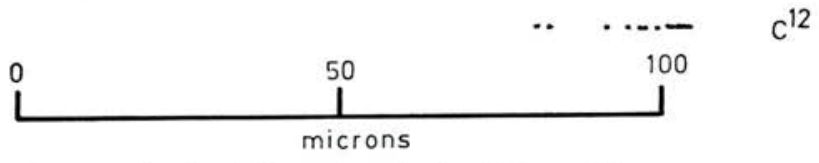

Fig. 16. - Attempted simulation of tracks in $K-2$ emulsion, as a 6-hit detector.

$K-2$

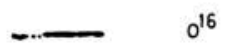

$E_{0}=7,60 \times 10^{6}$

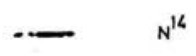

$c=10$

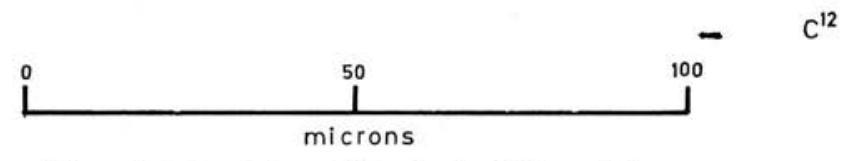

Fig. 17. - Attempted simulation of tracks in $K-2$ emulsion, as a 10-hit detector.

VOL. $10-\mathrm{N}^{\circ} 4$ 
photographed onto direct positive film, and are printed so that black dots appear on a white ground. The positions of the spots are computed from the theory. The program calculates the number of developed grains in long rectangular boxes parallel to the ion's path, whose length corresponds to a speed interval of $\Delta \beta=0.01$, and then distributes them according to a pseudorandom number program. The depth in the simulated emulsion, $d$ in Figure 4, must approximate the depth before processing of

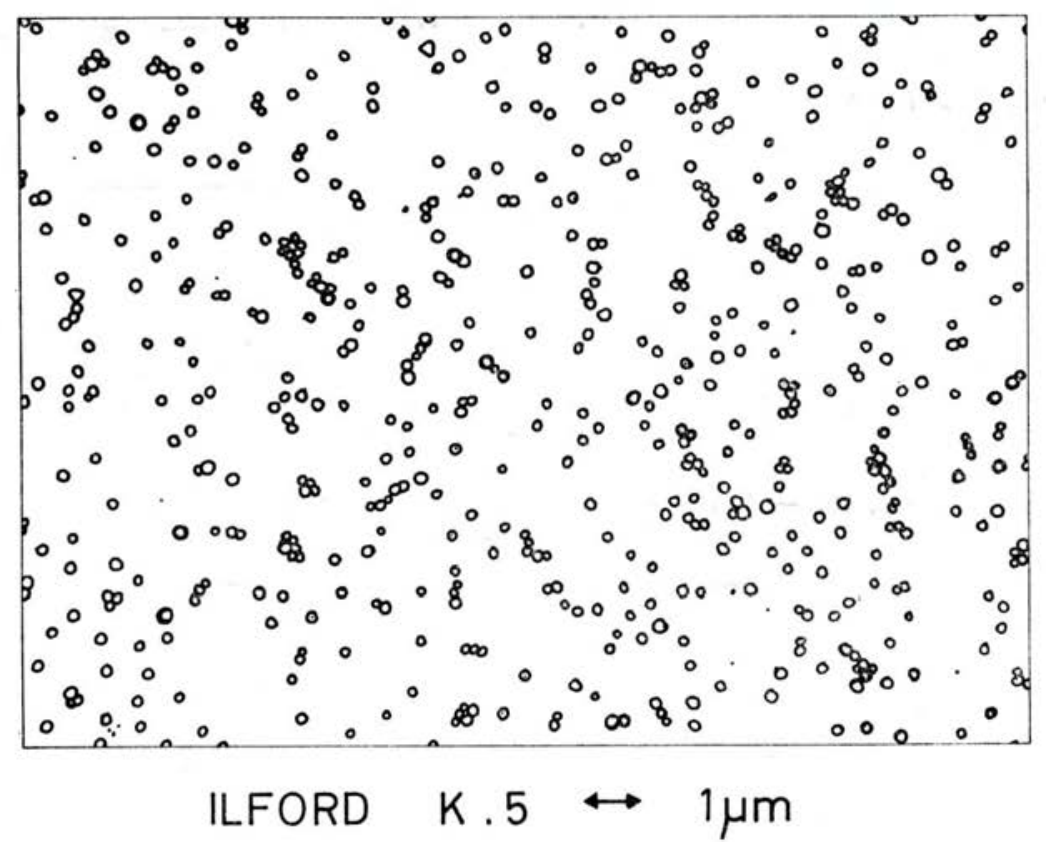

FIG. 18. - Grains in K. 5 emulsion. Courtesy J. Ehrlich.

that part of an emulsion layer which is in sharp focus in a microphofograph. Here we have taken $d=0.25 \mu \mathrm{m}$ to include the combined effects to depth of focus, shrinkage, and perception. The subvolumes $\left(A, B, B^{\prime}\right.$, $\left.C, C^{\prime}, \ldots\right)$ are divided into microboxes, as shown. We first find $\bar{E}$ in the microbox, then use it to find $P$, and then multiply this by the number of undeveloped grains in the microbox to yield the number of developed grains. We sum to find the number of developed grains in the subvolumes (which correspond to raster spacings in the visual display unit) and write on the face of the visual display unit. No attempt is made to group the developed grains into track-like structures, to represent delta-rays, nor is any attempt made to represent the scattering of the heavy ion. Tracks are then photographed in simulated $100 \mu \mathrm{m}$ segments. 
We have applied the track simulation procedure to analyze the radiosensitivity parameters of $K$-series emulsions exposed to heavy ions at $10 \mathrm{MeV} /$ nucleon [3], and processed (temperature cycle) in a developer of the Brussels type, after storage for 2 days at room temperature and $50 \mathrm{p}$. cent humidity. Differences from ion to ion arise from the different values of $z$ and $\beta$ at the same range. Differences from picture to picture are from different values of $m, c$, or $E_{0}$, but a single set of parameters describes all ions

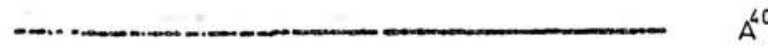

$K-2$

$E_{0}=2,30 \times 10^{7}$
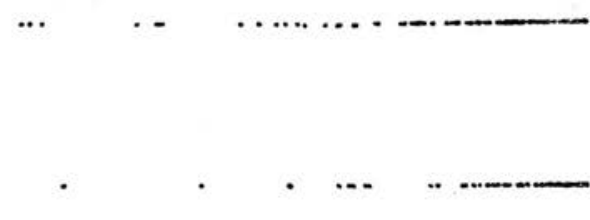

。 $2,30 \times 10^{7}$

$M=8$

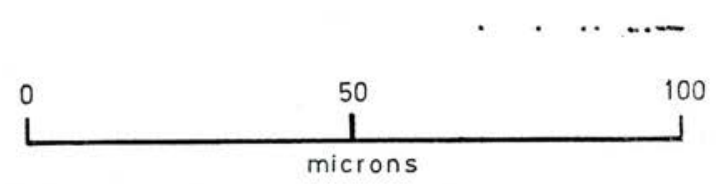

FIG. 19. - Attempted simulation of $K-2$ emulsion as an 8-target, single-hit per target detector.

in the simulation of a single emulsion picture. In all cases the tracks are of ${ }^{40} \mathrm{~A},{ }^{20} \mathrm{Ne},{ }^{16} \mathrm{O},{ }^{14} \mathrm{~N}$, and ${ }^{12} \mathrm{C}$, which enter the emulsion at high energy at left, and stop at right.

Track photographs in Ilford K.5 emulsion ( Fig. 5) are to be compared to simulations in Figure 6, with $c=1$ and $E_{0}=2 \times 10^{5} \mathrm{erg} / \mathrm{cm}^{3}$, in emulsion.

Track photographs in $K .0$ emulsion (Fig. 7) are to be compared with simulations in Figure 8, with $c=1$, and $E_{0}=2.4 \times 10^{7} \mathrm{erg} / \mathrm{cm}^{3}$, in emulsion.

Track photographs in $K-1$ emulsion (Fig. 9) are to be compared with simulations in Figure 10, with $c=1$ and $\mathrm{E}_{0}=4 \times 10^{7} \mathrm{erg} / \mathrm{cm}^{3}$, and in Figure 11, with $c=2$ and $E_{0}=1.3 \times 10^{7} \mathrm{erg} / \mathrm{cm}^{3}$, in emulsion. We judge these to be the best simulations we have been able to make with $c=1$ and $c=2$, and that

voL. $10-\mathrm{N}^{\circ} 4$ 
they demonstrate that $K-1$ emulsion is a 1-hit detector. At higher values of $c$ it is immediately obvious that the simulation does not match the observed track.

Track photographs in $K-2$ emulsion (Fig. 12) are compared with simulations for which $c=1$, and $E_{0}=1 \times 10^{8}$ and $4 \times 10^{8} \mathrm{erg} / \mathrm{cm}^{3}$, in Figures 13 and 14 , respectively. These are chosen to be a best possible approximation

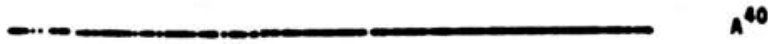

\section{$\boldsymbol{K}=\mathbf{2}$}

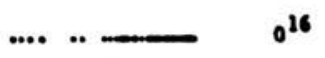

$E_{0}=1.75 \times 10^{7}$

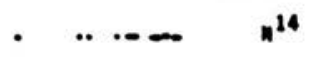

$\boldsymbol{n}=\boldsymbol{2 0}$
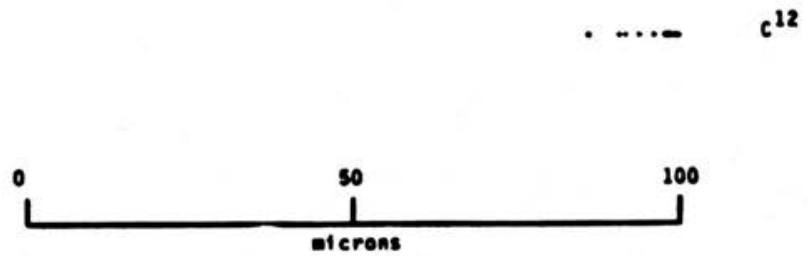

Fig. 20. - Attempted simulation of $K-2$ emulsion as a 20-target, single-hit per target detector.

(with the 1-hit assumption) to the ${ }^{40} \mathrm{~A}$ and to the ${ }^{12} \mathrm{C}$ experimental tracks, and clearly are an unsatisfactory simulation.

We have identified $K-2$ emulsion as an 8-hit detector, with $c=8$ and $E_{0}=8 \times 10^{6} \mathrm{erg} / \mathrm{cm}^{3}$, in emulsion, on the basis of Figure 15 , to be compared to Figure 12. A quite good simulation can be made with $c=9$, but simulations made with $c=6$ (Fig. 16) and $c=10$ (Fig. 17) are unsatisfactory.

It may be the uniformity of the emulsion grains in these nuclear emulsions, as shown for $K .5$ emulsion in Figure 18, that makes it possible to determine 
the value of $c$ so narrowly, as indicated by these simulations, where we find $c=8 \pm 1$, for Ilford $K-2$ emulsion as processed here.

Reasonably acceptable simulations of the tracks in $K-2$ emulsion may be made with the multi-target single-hit model, Equation (1), as shown in Figure 19, where $m=8$ and $E_{0}=2.3 \times 10^{7} \mathrm{erg} / \mathrm{cm}^{3}$, and in Figure 20, where $m=20$ and $E_{0}=1.75 \times 10^{7} \mathrm{erg} / \mathrm{cm}^{3}$. It is not possible to assign as narrow limits to $m$ as to $c$. This suggests that the functional form of the multi-hit model is a better approximation to the response of emulsion to gamma-rays than is the multi-target model.

\section{DISCUSSION}

The discovery that Ilford $K-2$ emulsion is a more-than-1-hit detector implies that its "RBE" must pass through a maximum at an appropriate value of the "LET" of a beam of incident particles, and that a maximal value of the RBE, greater than 1 , is not a uniquely biological property. If, as a rule-of-thumb, we take the RBE to be a maximum where about half the intersected grains are made developable, the maximum occurs for ${ }^{40} \mathrm{~A}$ ions at $7.4 \times 10^{3} \mathrm{MeV} \cdot \mathrm{g}^{-1} \cdot \mathrm{cm}^{2}$, where $\beta=0.15$, and the residual range is $109 \mu \mathrm{m}$, with a kinetic energy of $10.6 \mathrm{MeV} / \mathrm{amu}$. Here the grain count is calculated to be 3.5 grains $/ \mu \mathrm{m}$. At a fluence of $10^{6}$ ions $/ \mathrm{cm}^{2}$ where the dose in emulsion is 120 rads, and where the contribution to gamma-kill from overlapping deltarays from different ions is negligible, the " $\mathrm{RBE}$ " relative to gamma-rays is calculated to be about 300 .

We must expect to see in emulsion, as we cannot see in biological systems, the effects of very low levels of radiation, and the transition in effect from purely ion-kill to a mixture of ion-kill and gamma-kill. Though single energetic electrons, protons, alpha-particles make no grains developable in $K-2$ emulsion, beams of these particles must blacken the emulsion.

Since there is no evidence of internal sensitive sites in emulsion grains, as there is in biological cells, we anticipate that 3 radiosensitivity parameters, $E_{0}, a_{0}, c$ will suffice to describe their properties, in place of the 4 parameters, $E_{0}, k, \sigma_{0}, m$, required to describe cells.

Both $E_{0}$ and $c$ can be expected to be affected by manufacture, by processing, and by fading.

The Ilford $K$-series of emulsions is manufactured from a parent stock called $K .0$, increasingly sensitized to become $K .1$ to $K .5$, and desensitized to become $K-1, K-2, K-3$. We have no data for $K-3$ emulsion, but otherwise in this series the most striking change is the abrupt increase in hittedness from $K-1$ to $K-2$, resulting in a qualitatively different detector.

There are suggestions of more-than-1-hittedness in other emulsions and with other processing procedures. The incorporation of sodium rhodanide (NIKFI T-1) produced a sharp discrimination between the tracks of recoil protons and alpha particles, from neutron irradiation [1]. The use of $\mathrm{pH}$ buffers in the developer yielded a discrimination between alpha particles, fission fragments, and $\mathrm{Ne}$ ions in $K-2$ and $K-3$ emulsions [1], and 
in Kodak NT2a emulsion [15]. There are other studies of discriminating development $[18,19]$. It has been suggested that these developers tend to bleach the smaller latent image sites, and only crystals through which several electrons had passed would become developable.

It seems reasonable to anticipate that further investigation will yield quantitative values of the radiosensitivity parameters, for different emulsionprocessing techniques, and that the theory will be of assistance in selecting emulsions whose properties match those of biological cells and tissues, in regard to RBE-LET variations, to be useful in both radioprotection and radiation therapy, and to serve as a modeling system for the study of dose-rate and low-level effects in radiobiology.

\section{REFERENCES}

[1] Barkas W. H. Nuclear Research Emulsions 1, New York, Academic Press, 1963.

[2] BeCKer K. Fortschr. Geb. Rontgenstr. Nuclearmed., 1961, 95, 694-703; 837-847.

[3] Benton E. V., Tochilin E. Health Physics, 1966, 12, 49-52.

[4] Butrs J. J., Katz R. Rad. Res., 1967, 30, 855-871.

[5] Dertinger H., Jung H. Molecular Radiation Biology, New York, Springer Verlag, 1970.

[6] Digby N., Firth K., Hercock R. J. J. Photogr. Sci., 1953, 1, 194-208.

[7] Furtak T. E., Katz R. Rad. Effects, 1971, 11, 195-199.

[8] Golden R., Tochilin E. Health Physics, 1959, 2, 199-206.

[9] Herz R. H. Photographic Action of Ionizing Radiations, New York, Wiley-Interscience, 1969.

[10] Jensen M., Larsson L., Mathiesen O., Rosander R. Report LUIP-CR-74-07, 1974, Department of Physics, University of Lund, S-223 62, Lund, Sweden.

[11] Jensen M., Mathiesen O. Report LUIP-CR-75-03, 1975, Department of Physics, University of Lund, S-223 62, Lund, Sweden.

[12] Katz R., Коветісн E. J. Phys. Rev., 1969, 186, 344-351.

[13] Katz R., Коветісн E. J. Nucl. Instr. Methods, 1970, 79, 320-324.

[14] Katz R., Sharma S. C., Homayoonfar M. In Topics in Radiation Dosimetry, Supplement 1 (F. H. Attix, Ed.) New York, Academic Press, 1972.

[15] Stevens G. W. W. In Photographic Sensitivity (J. W. Mitchell, Ed.) London, Butterworth, 1951.

[16] Tochilin E., Golden R. Health Physics, 1961, 4, 244-249.

[17] Valentine R. C. Adv. Opt. Electr. Microscopy, 1966, 1, 180-203.

[18] Zakharov V. J., Kocherov N. P., Novikova N. R., Perfilov N. A. 8th Int. Conf. on Nuclear Photography, Bucharest, 1972 (M. Nicolae, Ed.).

[19] De Carvalho H. G. In Progress in Nuclear Techniques and Instrumentation, Vol. I (F. J. M. Farley, Ed.) Amsterdam, North-Holland, 1965. 